\title{
DE LA PEDAGOGÍA A LA “PODAGOGÍA”. EL PODCASTING EN LA EDUCACIÓN SUPERIOR
}

\author{
Teresa Piñeiro Otero. Universidad da Coruña (España)
}

\section{Introducción}

La irrupción de Internet y de las nuevas tecnologías de la información y de la comunicación, las llamadas TIC, ha adquirido un papel determinante en el contexto actual, con especial relevancia en el ámbito educativo. En efecto, en los últimos años, la implantación de las TIC ha constituido el eje prioritario de las políticas educativas de las diferentes instituciones -autonómicas, centrales y europeas- con competencias en la materia.

En la apuesta por la flexibilidad de las instituciones de Educación Superior y la respuesta a las nuevas demandas del aprendizaje permanente, resulta de especial relevancia la incorporación de la Web 2.0 a las metodologías didácticas. Esta «nueva» concepción de la web ofrece múltiples herramientas que, utilizadas con finalidad educativa, pueden enriquecer el proceso de enseñanza-aprendizaje.

Entre las herramientas de la Web 2.0 que se han incorporado con éxito al ámbito de la educación resultan de particular interés los podcast.

Un podcast es un archivo digital de sonido -aunque también existen podcast de vídeo- distribuido a través de unawebsite o un repositorio online, que permite su escucha en línea o su descarga para su consumo offline(Borges, 2009: pág. 17).

La aparición de esta tipología de archivos de audio sindicado constituyó el fin de un proceso que, siguiendo a Tenorio $(2008,9)$, comenzó en el año 2000. En este año David Winer desarrolló una nueva etiqueta para la versión 0.92 de RSS «enclosure» que permitió la sindicación del primer archivo de audio. En 2004 el propio Winer puso fin a este proceso con la creación, junto a Adam Curry (DJ de MTV) de una aplicación informática que posibilitó la descarga automática de programas de radio online en el iPod.

En este sentido se puede definir podcasting como una forma de distribución de archivos de audio que "A través de la suscripción de un feed RSS, y con la ayuda de un programa específico, se puede descargar de forma automática en el ordenador 0 iPod" o cualquier otro dispositivo multimedia (Moura y Carvalho, 2006: pág. 88).

Es, precisamente, esta posibilidad de sindicación, el elemento diferencial de los podcast respecto a cualquier otro tipo de archivos de sonido web. 
Debido a su esencia fundamentalmente sonora, la inclusión del podcasting en las metodologías didácticas se ha beneficiado de aquellas potencialidades asociadas al audio. Algunos de sus beneficios son la predisposición positiva de los alumnos hacia los materiales sonoros, la posibilidad de revisión de dichos materiales, la recepción de indicaciones para el desarrollo de prácticas, la escucha de disertaciones de expertos en la materia o el incremento de la atención ante una voz conocida y respetada (Durbridge en Salmon y Nie, 2008: pág. 4).

Más allá de sus potencialidades como herramienta didáctica de base sonora, la integración de los podcast en las estrategias educativas se ha relacionado con determinados efectos de carácter positivo como la mayor atracción del estudiantado por los contenidos de las materias, o el fomento de su imaginación.

El empleo del podcasting en el ámbito de la Educación Superior se ha visto favorecido por tres aspectos concretos:

- la importante penetración de la Red

- la accesibilidad del software destinado a la edición online de archivos sonoros y

- la incipiente implantación y multiplicación de dispositivos portátiles (iPod, MP3, PDA, smartphones, etc.) idóneos para el consumo de archivos sonoros (EDUCAUSE, 2006).

Además de dichos aspectos, de carácter fundamentalmente tecnológico, resulta destacable otra cuestión con implicación directa en la aceptación de los podcast: la creación y participación de comunidades online (Piñeiro, 2009).

En efecto, siguiendo a Edirisngha y Salmon (2007), la adopción de las aplicaciones de la Web 2.0 ha permitido a los usuarios -estudiantes y profesores- capturar, generar y compartir contenido, creando verdaderas comunidades educativas que han contribuido a la popularidad de esta herramienta.

\section{Objetivos}

Para comprender la relevancia que ha adquirido el mediacasting en el ámbito de la educación, especialmente en lo que se refiere a los podcast de audio, resulta importante conocer dicho fenómeno así como sus potencialidades para el proceso de enseñanza-aprendizaje.

De este modo, el objetivo principal de este trabajo es el de efectuar una aproximación al concepto de podcast, sus principales manifestaciones y potencialidades en el ámbito de la Educación Superior.

Como objetivo secundario se ha planteado señalar las principales iniciativas de podcasting didáctico desarrolladas en el ámbito de la Educación Superior en general y, más concretamente, aquellas desarrolladas en el ámbito académico español.

\section{Metodología}

Para lograr los objetivos planteados se ha efectuado una revisión bibliográfica sobre 
el estado de la cuestión del podcasting en el contexto de la Educación Superior.

Asimismo, para conocer las principales iniciativas desarrolladas en el ámbito español se complementó dicha revisión bibliográfica con el análisis de su presencia en los websites de las universidades, y su enlace a portales externos, que actúan como repositorios de estos contenidos.

\section{Podcasting educativo}

La eclosión y expansión del fenómeno podcasting ha propiciado su proyección en diversos ámbitos, más allá del estrictamente radiofónico. En la actualidad resulta fácil encontrar podcast de diferentes temáticas con una multiplicidad de objetivos, entre los que destacan el educativo-formativo.

En este contexto se ha definido podcasting como el proceso de capturar un evento sonoro -canción, discurso o una mezcla de todos- y publicarlo como un archivo de audio digital, en un website o blog, siguiendo una estructura de datos o feed (Meng, 2005).

Más concretamente, Solano y Sánchez (2010: pág. 128) han definido los podcasteducativos como un:

medio didáctico que supone la existencia de un archivo sonoro con contenidos educativos y que ha sido creado a partir de un proceso de planificación didáctica. Puede ser elaborado por un docente, por un alumno, por una empresa o institución.

Esta definición incide en el hecho de que un podcast educativo no es solamente un archivo de audio puesto a disposición de una comunidad educativa, sino que debe estar vinculado a una planificación didáctica, con unos objetivos pedagógicos claros. Una vez "subidos" a la Red, los estudiantes-usuarios solamente deben acceder al podcast en la página o repositorio web una vez, suscribiéndose a su contenido. A partir de este primer acceso, el estudiante recibirá directamente en su ordenador las diferentes actualizaciones del podcast, ya para escucharlo desde éste, ya para transferirlo a cualquier tipo de dispositivo portátil (Kaplan-Leiserson, 2005).

Precisamente, una de las características que han influido positivamente en la expansión vivida por los podcast ha sido la posibilidad de suscribirse a los archivos de interés vía RSS (Real Simple Syndication) o Atom, y su «portabilidad».

Se puede tener acceso a podcast en cualquier dispositivo digital que permita la reproducción de archivos de audio y multimedia como es el caso de los netbook, MP3, teléfonos móviles de tercera generación (3G), smartphones, PDA (Personal Digital Assistant), PSP (Play Station Portable), etc.

En el ámbito de la cultura de la portabilidad (Kichinhesvky, 2009) estos dispositivos digitales se multiplican en los bolsillos de los universitarios, pertenecientes a las generaciones de nativos digitales (Presnky, 2001).

Como señalan Salmon y Nie (2008): 
(...) cientos de estudiantes caminan alrededor del campus, se sientan en los autobuses o se tumban en la hierba del parque, con los reproductores MP3 'pegados' a sus orejas. Lo que en un principio podría parecer una aproximación un poco "techie" a la música de repente puede adquirir un gran valor y un bajo coste para la educación".

El reto, en este caso, es el de crear contenidos educativos que se perciban de utilidad para lograr conquistar espacios y momentos, gigas y minutos, anteriormente reservados para el ocio.

\subsection{Potencialidades para la educación}

Pese a lo novedoso del fenómeno-apenas han pasado seis años de la creación del primer podcast- existe un creciente número de investigaciones en torno al uso del podcasting en el contexto académico. Estas investigaciones han destacado las posibilidades de los podcast en el proceso de enseñanza-aprendizaje, focalizando su atención en los efectos positivos de su empleo en los estudiante como el enriquecimiento de la experiencia de aprendizaje (Boulos, Maramba y Wheeler, 2006), la potenciación de la atención del estudiante y su reflexión (Baird y Fisher, 2006), el incremento de satisfacción (Miller y Piller, 2005) o, incluso, la reducción de la ansiedad (Chan y Lee, 2005).

Otro aspecto positivo, siguiendo a Moura y Carvalho (2006) es el de permitir al profesor sintonizar con los estudiantes de hoy, los nativos digitales. Precisamente, la incorporación del podcasting a las estrategias educativas, facilita el proceso de enseñanza-aprendizaje de estos nativos digitales.

Siguiendo a Borges (2009: pág. 35-36)el empleo del podcasting en el contexto educativo favorece:

- Potencialidades cognitivas. La utilización educativa de los podcast potencian las competencias de los estudiantes en comunicación y relación personal, aprendizaje colaborativo, así como en la interpretación, análisis, selección y difusión de contenidos.

- Implicación del estudiante. Realizar actividades con podcast favorece el aprendizaje autónomo.

- Autogestión del estudiante. Los podcast contribuyen a que el estudiante planifique su trabajo, dada su esencia asíncrona, para su escucha fuera del aula, en tiempos muertos y de ocio.

- Disposición de las indicaciones del profesor. Los podcast permiten la escucha, en cualquier momento y lugar, de aclaraciones o explicaciones respecto al material de aula.

- Continuidad en el estudio. Los podcast pueden contribuir a la continuidad del estudiante, a gestionar y utilizar sus contenidos con cierta regularidad.

- Comprensión. Dada las posibilidades de repetición, los podcast facilitan la comprensión de determinados contenidos, al tiempo que refuerzan dicho aprendizaje.

- Reducción de la ansiedad. Los podcast pueden disminuir la ansiedad de los estudiantes ante la preocupación por los contenidos de una determinada materia o su evaluación, al poder revisarlos siempre y cuando quiera. 
Si bien no es la primera vez que se incorporan metodologías educativas basadas en audio (desde los casetes analógicos, hasta los archivos de audio online, pasando por la radio), sí resultan de especial interés los podcast por facilitar su consumo individual y la suscripción. En efecto, el estudiante que opte por el uso de podcatchers como iTunes o iPodder $^{1}$ puede descargar automáticamente los contenidos de interés en su ordenador, lo que facilita el acceso, consumo y -por tanto- el enriquecimiento del proceso de enseñanza-aprendizaje (Bennington, 2007).

Como señalan Woodland y Klass $(2005,5)$, "El podcasting no es simplemente una nueva forma de distribuir grabaciones de audio, es una forma de expresión, interacción y creación de comunidad". De hecho, aunque resulte aparentemente contradictorio, la incorporación del podcasting en el contexto de la Educación Superior permite el desarrollo de un aprendizaje, a la vez autónomo y colaborativo.

\subsection{Tipologías de 'podcast' didácticos}

Siguiendo a Salmon y otros (2007), la utilización de los podcast en el proceso de enseñanza-aprendizaje puede atender a diferentes propósitos pedagógicos.

La incorporación del podcasting al ámbito de la educación no ha dado lugar al desarrollo de una única metodología didáctica. Al contrario, la multiplicidad de características y objetivos que presenta el empleo de dichos archivos, ha llevado a establecer diferentes tipologías de podcast.

A la distinción genérica entre los audiocast o podcast de audio, y vodcast o podcast de vídeo, resulta preciso sumar otras tipologías de podcasting educativo.

En este sentido Hürst y Waizenegger (2006) emplean términos como learncastingopodagogy, para referirse a las metodologías didácticas basadas en podcast o con apoyo de estos. Por su parte, si el empleo de estos archivos se concreta al ámbito docente, se puede hablar de profcasting.

Además de dichas denominaciones, el análisis bibliográfico ha permitido determinar otros términos con menor incidencia en el corpus teórico, dada su referencia a realidades muy concretas. Este es el caso -por ejemplo- del lecturecasting, una tipología de podcast que vehicula la grabación de clases o conferencias.

\section{Principales experiencias desarrolladas}

La primera iniciativa del empleo de podcasting, con finalidad didáctica, suele atribuirse al College Jogakuin de Osaka (Japón). En 2004 esta institución introdujo los podcast en las metodologías didácticas para la enseñanza de inglés como lengua extranjera (Mochizuki citado en Solano y Vera, 2010).

Una innovación didáctica que se desarrolló prácticamente de forma conjunta al lanzamiento y eclosión del podcasting.

\footnotetext{
${ }^{1} \mathrm{U}$ otro tipo de podcachers gratuitos como Juice, jPodder, gPodder o PPR.
} 
Entre las primeras instituciones que experimentaron las potencialidades de los podcast en el proceso de enseñanza-aprendizaje destaca la Universidad de Duke.

La puesta en marcha de la Duke Digital Initiative implicó el desarrollo de contenidos didácticos en formato podcasting, entre los que se encontraba material de estudio, grabaciones de clase, prácticas de campo u otros contenidos complementarios para las materias ${ }^{2}$. Además de la creación de dichos materiales, se distribuyeron iPods entre el alumnado, para facilitar el acceso y consumo de estos contenidos didácticos (Belanguer, 2005).

La Universidad de Duke también detenta el mérito de ser la primera -siguiendo a Lankshear y Knobel (2006: pág. 176)- en crear y alojar podcast de un congreso universitario ${ }^{3}$.

También la Harvard Medical School han desarrollado estrategias educativas basadas en iPod \& podcast, para e enriquecer el proceso de enseñanza-aprendizaje (Moura y Carvalho, 2006).

Otra de las universidades pioneras en la implementación de metodologías didácticas basadas podcast fue la Universidad de Stanford; universidad que cuenta con un iTunes institucional ${ }^{4}$, al que pueden suscribirse los estudiantes y descargar grabaciones de clases u otro material complementario, para su escucha en el iPod.

Entre las experiencias de podcasting en el ámbito de la Educación Superior resulta de particular relevancia la plataforma impulsada por Apple, iTunes University. La empresa, vinculada al podcasting desde sus orígenes, puso en marcha -junto a diversas universidades de Estados Unidos- este repositorio académico que facilita a los estudiantes diversos contenidos docentes en formato podcast (Moura y Carvalho, 2006).

En el contexto europeo destaca la puesta en marcha del Informal Mobile Podcasting and Learning Adaptation (IMPALA) ${ }^{5}$. Un proyecto educativo, desarrollado en Reino Unido, que contó con la participación de 20 profesores universitarios, y 500 estudiantes de diferentes áreas y disciplinas.

El IMPALA, dio comienzo en mayo de 2006, como un programa piloto en una de las titulaciones en ingeniería de la Universidad de Leicester. Este programa permitió subrayar las potencialidades profcasting para el enriquecimiento del proceso de enseñanza-aprendizaje de una manera lúdica, accesible y asequible.

Más allá de estas primeras iniciativas, el verdadero impulso al podcasting en el ámbito de la Educación Superior tuvo lugar a partir del año 2006. Año en el que, siguiendo a Salmon y Nie (2008) se extiende el empleo de esta herramienta.

\footnotetext{
${ }^{2}$ Se puede acceder a ellos en la dirección www.duke.edu/ddi/ (10/7/2010).

3 Duke Podcast Simposium, celebrado el 27-28 de septiembre y disponible en la dirección www.isis. duke.edu/events/podcasting/bios.html (16/8/2010).

${ }_{5}^{4}$ La dirección es http://itunes.stanford.edu/ (07/8/2010).

${ }^{5}$ www.impala.ac.uk (10/8/2010).
} 


\subsection{El podcasting didáctico en la universidad española}

En el ámbito español, la implantación de los podcast en la Educación Superior se desarrolló tardíamente si se atiende a las primeras iniciativas de este tipo en Estados Unidos. La propia creación de un canal en iTunes- $U$ para las instituciones académicas españolas no tuvo lugar hasta el año 2009, cuando ya habían transcurrido dos años de la creación de dicha plataforma.

En la actualidad (2010) la plataforma educativa de Apple cuenta con la presencia de seis instituciones españolas de Educación Superior: IE Business School, Universidad de Alicante, Universidad de Valladolid, Universidad Politécnica de Madrid, Universidad Rey Juan Carlos y Universidad de Vigo.

Al margen de este repositorio académico, en el ámbito de la universidad española, se pueden señalar otras iniciativas didácticas basadas en la utilización de podcast.

Tabla 1. Universidades españolas con proyectos de Podcast educativos

\begin{tabular}{|l|l|}
\hline \multicolumn{1}{|c|}{ Institución } & \multicolumn{1}{c|}{ WEB } \\
\hline Universidad de Navarra & http://www.unav.es/alumni/podcast/ \\
\hline Universidad Europea de Madrid & http://www.podcastuem.com/ \\
\hline Universidad de Pamplona & http://www.podcast.unipamplona.edu.co/ \\
\hline Universidad Carlos III de Madrid & http://arca.rediris.es/doc.php?dmod=user \\
\hline
\end{tabular}

Fuente: Elaboración propia.

Entre estas iniciativas resulta especialmente relevante esta última, el Proyecto ARCA, dado que se configura como un repositorio global que trasciende el ámbito concreto de la universidad de origen (Universidad Carlos III).

El proyecto ARCA, acrónimo de "Agregador RSS para la Comunidad Académica", constituye una plataforma de federación de contenidos multimedia creados por las instituciones integrantes de la red académica RedIRIS.

Entre sus objetivos se encuentra el de vehicular y difundir, entre la comunidad académica, aquellos contenidos generados por docentes e instituciones educativas superiores, que puedan resultar útiles tanto para el proceso de enseñanzaaprendizaje como para aportar información de interés. Una información, en su mayoría de carácter institucional, que llega a solapar los contenidos didácticos propiamente dichos.

En definitiva, pese a las potencialidades del podcasting para la optimización del proceso de enseñanza-aprendizaje, su empleo en el ámbito de la Educación Superior española resulta todavía testimonial. 


\section{A modo de conclusión}

La aproximación llevada a cabo en torno al fenómeno podcasting y su incorporación a las metodologías didácticas ha puesto de relevancia sus potencialidades para la Educación Superior.

El desarrollo de contenidos de carácter educativo en formato podcast -ya se trate de grabaciones de las aulas teóricas, aclaraciones sobre éstas, instrucciones para prácticas, disertaciones de expertos, etc.- favorece la optimización del proceso de enseñanza-aprendizaje. Proceso que, una vez liberado de cualquier barrera espaciotemporal, permite la recuperación de momentos y lugares otrora perdidos para la educación.

La cuestión, en este caso, radica en si los estudiantes estarían dispuestos a emplear para la educación sus dispositivos de entretenimiento y sus burbujas de ocio intersticial (Igarza, 2009). En efecto, como señala Borges (2009), el primer requisito para incorporar los podcast al proceso de enseñanza-aprendizaje es el de compartir, con los contenidos educativos, el espacio en la memoria de aquellos dispositivos móviles habitualmente asociados a consumos lúdicos.

En cualquier caso, aunque cada vez son más las instituciones de Educación Superior que apuestan por los podcast en sus metodologías didácticas, todavía se debe hablar de iniciativas concretas y aisladas. Esta circunstancia supone una infrautilización de las posibilidades que la penetración y multiplicación de los dispositivos portátiles brindan para el desarrollo de un aprendizaje always y everywere.

\section{Bibliografía}

\section{Libros:}

BORGES, F. (2009). Profcast: Aprender y enseñar con podcast. Barcelona: Editorial UOC.

CASTAÑO, C.; Maiz, I.; PALACIO, G. y VILARROEL, J.D. (2008). Prácticas educativas en entornos web 2.0. Madrid: Síntesis.

EDUCAUSE (2006). The Horizon Report, 2006. Austin: The New Media.

GALLEGO, J.I. (2010). Podcasting. Nuevos modelos de distribución para los contenidos sonoros. Barcelona: UOCpress.

IGARZA, R. (2009). Burbujas de ocio: nuevas formas de consumo cultural. Buenos Aires: La Crujía.

LANKSHEAR, C. y KNOBEL, M. (2006). New literacies: Everyday Practices \& Classroom learning. Nueva York: McGraw-Hill Education.

MILLER, M. y PILLER, M. (2005). Principal factors of an audio reading delivery 
mechanism - evaluating educational use of the iPod. En Kommers, P. y Richards, G. (Eds.), Proceedings of World Conference on Educational Multimedia, Hypermedia and Telecommunications 2005. Chesapeake: AACE. pp. $260-267$

MOURA, A. y CARVALHO, A. A. (2006). Podcast: Uma ferramenta para usar dentro e fora da sala de aula. En José, R. y Baquero, B. (eds): Proceedings of the Conference on Mobile and Ubiquitous Systems. Guimarães: Universidade do Minho. pp.155-158.

SALMON, G. y NIE, M. (2008). Doubling the life of iPods. En Salmon, G. y Palitha, E. Podcasting for learning in universities.Reino Unido: McGrawhill.

TENORIO, I. (2008). La nueva radio. Manual completo del radiofonista moderno.Barcelona: Marcombo.

WOODLAND, M. y KLASS, D. (2005). Podcast solutions: the complete guide to podcasting. Nueva York: Friendsoft.

\section{Artículos en revistas:}

BENNIGTON, A. (2007). Stick it in your ear: Keeping current with podcasts, en Online, vol. 31, no 3, p. 30-32.

INFANTE, I. (2006). Emissões Livres, en Revista Exame Informática, no 130, p.106109.

KISCHINHESKY, M. (2009). Cultura da portabilidade - Novos usos da rádio e sociabilidades em mídia sonora, en Observatorio (OBS*) Journal, no 8, p. 223238.

MOURA, A. y CARVALHO, A. A. (2006). Podcast: Potencialidades na Educação, en Prisma.com, nำ 3, p. 88-120.

PRENSKY, M. (2001). Digital natives, digital immigrants part 1, en On the horizon, vol. $9, n^{\circ} 5$, p. 1-6.

PIÑEIRO OTERO, Teresa (2009). Podcasting en la educación superior. Una aproximación. en Revista de la SEECI, № 18, p. 129 -147.

SOLANO FERNÁNDEZ, I. M. y Sánchez Vera, M. M. (2010). Aprendiendo en cualquier lugar: el podcast educativo, en Pixel-Bit. Revista de Medios y Educación, no 36, p. 125-139.

\section{Recursos electrónicos:}

BELANGER, Y. (2005). Duke University iPod first year experience final evaluation report. Consultado el 18 de marzo de 2010. Disponible en línea en: http://cit.duke.edu/pdf/ipod initiative 04 05.pdf 
HAMMERSLEY, B. (2004). Audible revolution. The Guardian, 12 de febrero de 2004. Consultado el 10 de marzo de 2010. Disponible en línea en: www.guardian.co.uk/media/2004/feb/12/broadcasting.digitalmedia

HÜRST, W. y WAIZENEGGER, W. (2006). An overview of different approaches for lecture casting. Proceedings of IADIS International Conference on Mobile Learning 2006, July 2006. Consultado el 18 de marzo de 2010. Disponible en línea en: www.iadis.net/dl/final uploads/200605F008.pdf

KAPLAN-LEISERSON, E. (2005). Trend: Podcasting in academic and corporate learning. Consultado el 12 de enero de 2010. Disponible en línea en Learning Circuits: www.astd.org/LC/2005/0605 kaplan.htm

MENG, P. (2005). Podcasting and vodcasting: A white paper. Definitions, discussions, and implications. Consultado el 12 de enero de 2010. Disponible en línea

en: http://edmarketing.apple.com/adcinstitute/wpcontent/Missouri Podcasting Whit e Paper.pdf 